\title{
The Prognosis of Lung Cancer With Different Metastatic Patterns
}

\author{
SHINICHIRO OKAUCHI ${ }^{1}$, HIROKO WATANABE ${ }^{2}$, HIDEYASU YAMADA $^{3}$, SHINYA SATO $^{4}$, \\ KUNIHIKO MIYAZAKI ${ }^{4}$, TAKAHIDE KODAMA ${ }^{4}$, HIROAKI SATOH ${ }^{1}$ and NOBUYUKI HIZAWA ${ }^{5}$ \\ ${ }^{1}$ Division of Respiratory Medicine, Mito Medical Center, \\ University of Tsukuba-Mito Kyodo General Hospital, Mito, Japan; \\ ${ }^{2}$ Division of Respiratory Medicine, Tsukuba Kinmen General Hospital, Tsukuba, Japan; \\ ${ }^{3}$ Division of Respiratory Medicine, Hitachinaka Medical Center, \\ University of Tsukuba-Hitachinaka General Hospital, Hitachinaka, Japan; \\ ${ }^{4}$ Division of Respiratory Medicine, Ryugasaki Saiseikai General Hospital, Ryugasaki, Japan; \\ ${ }^{5}$ Division of Respiratory Medicine, Faculty of Medicine, University of Tsukuba, Tsukuba, Japan
}

\begin{abstract}
Background/Aim: Distant organ metastases do not occur at random in lung cancer. A retrospective study was conducted in order to evaluate 1) what kinds of metastatic patterns exist in three different types of lung cancer, and 2) whether metastatic patterns affected prognosis in the different types of lung cancer. Patients and Methods: Data were collected from all consecutive patients with diagnosed lung cancer between April 2009 and October 2018 in our hospitals. Cluster analysis was performed to classify patients. Kaplan-Meier analysis, log-rank test, and Cox proportional hazards model were used. Results: Epidermal growth factor-mutated adenocarcinoma, small cell lung cancer, and squamous cell lung cancer had different 'metastatic patterns', survival, and unfavorable prognostic factors, respectively. Conclusion: There might be different metastatic patterns, survival, and unfavorable prognostic factors in each pathological and genetic type of lung cancer. It is worthwhile carrying out diagnostic imaging and treatment considering information on metastatic patterns.
\end{abstract}

Distant organ metastases do not occur at random, and they do not occur uniformly, even if there is hematogenous and lymphogenous metastasis (1-4). It is clear that metastases located near the primary site are more frequent than sites

Correspondence to: Hiroaki Satoh, MD, Ph.D., Division of Respiratory Medicine, Mito Medical Center, University of Tsukuba-Mito Kyodo General Hospital, Miya-macho 3-2-7, Mito-city, Ibaraki, 310-0015, Japan. Tel: +81 292312371, e-mail: hirosato@md.tsukuba.ac.jp

Key Words: Cluster analysis, prognosis, lung cancer, metastatic pattern. distant from the primary site considering hematogenous and lymphogenous routes (1-3). Patients with solitary metastasis are rare (5), and the majority of patients have metastases in several organs at the time of diagnosis or during their clinical course (6). However, patients may develop solitary brain and metacarpal bone metastases $(7,8)$. We have been interested in distant organ metastases from lung cancer $(4,8-11)$, and if it turns out that certain metastatic patterns are associated with a good or poor prognosis, there is an opportunity to implement improved examination and treatment; appropriate medical resources could be efficiently introduced that would be clinically meaningful. In lung cancer, treatment types, response to treatment, and prognosis differ by tissue type. In lung cancer patients with well-defined driver genes, specific treatments exist, and there are subtypes for which long-term prognosis can be expected. When examining the relationship between metastasis patterns and prognosis, it should be considered according to subtypes classified by tissue type and driver gene.

Here, we conducted a study with the aim of obtaining answers to these two questions: 1) what kinds of metastatic patterns exist in each group of lung cancer patients, and 2) whether metastatic patterns affect prognosis in the different types of lung cancer.

\section{Patients and Methods}

Patients. Patients who presented with pathologically diagnosed lung cancer between April 2009 and October 2018 at three tertiary hospitals in Japan; University of Tsukuba Mito Medical Center-Mito Kyodo General Hospital, Ryugasaki Saiseikai General Hospital, and Tsukuba Kinmen General Hospital, were identified retrospectively via computerized searches of tumor registry data. In addition, we included all consecutive patients diagnosed with these three lung cancer types, stages IA-IIIC, in order to compare survival with 
different metastatic clusters. Medical record information from diagnostic imaging, including chest CT, brain MRI or enhanced head CT, bone scan and ultrasonography and/or CT of the abdomen, was used to identify the location of metastatic tumors. Information on distant metastases was collected in detail, with the most common metastatic sites being lung, bone, brain, liver, adrenal gland and distant lymph nodes. Clinical data regarding age, gender, smoking habit, primary site of lung cancer, maximum diameter of the primary tumor, and $\mathrm{N}$-stage of lung cancer were also collected.

This study was approved by the institutional ethics committee of each Hospital (Project approval number: NO16-66-NO18-17). Written comprehensive informed consent at the time of admission for obtaining pathological specimens was obtained from each patient.

Statistical analysis. Cluster analysis was performed to classify patients (12). Briefly, pre-clusters to reduce the size of the matrix that contained the distances between all possible pairs of cases were performed. Then, a standard hierarchical clustering algorithm was applied to the pre-clusters to explore a range of solutions with different numbers of clusters. At this point, hierarchical cluster analysis was performed using Ward's method to generate a dendrogram for estimation of the number of likely clusters within the population. Cluster boundaries were defined by large differences between successive fusion levels. At each cluster, samples were merged into larger clusters to minimize the within-cluster sum of squares, or to maximize the between-cluster sum of squares in Euclidean distance. Variables for cluster analysis included the common metastatic sites described above. The type of EGFR mutation was also included as a variable in patients with $E G F R$ mutant tumors. Statistical analyses were performed using BellCurve for Excel (version 3.0). Differences in proportions between two and among three independent groups were compared using the chisquare test.

Each cluster name used was that of the metastatic organ which met the following requirement: the cluster name was the metastatic organ(s) that more than half of the patients were assigned to in each cluster.

Kaplan-Meier analysis was used to assess survival curves, and the log-rank test was used in the univariate analysis to evaluate the statistical significance of survival between two groups. The survival time was defined as the interval (in months) between the date of initial therapy or supportive care until the date of death or the date of the last follow-up. Significant variables identified in the univariate analysis were included in the multivariate survival analysis using the Cox proportional hazards model to investigate the effects of clinicopathological factors, including metastatic patterns on survival. $p<0.05$ was considered a statistically significant difference.

\section{Results}

Patients. All 313 consecutive and pathologically diagnosed lung cancer patients with metastatic disease (99 patients with EGFR-mutated adenocarcinoma, 127 patients with SCLC, and 87 patients with squamous cell carcinoma) were included in the study. The most common metastatic sites were the lung, bone, brain, liver, and adrenal gland. We included 228 consecutive lung cancer patients with stage IA to IIIC in the
Table I. Characteristics of patients with EGFR mutated adenocarcinoma, those with small cell lung cancer, and those with squamous cell lung cancer.

\begin{tabular}{lccc}
\hline & Adeno & Small & Squamous \\
\hline $\begin{array}{l}\text { Metastatic } \\
\text { Age (median, range), }\end{array}$ & $71.4,44-89$ & $72.4,52-96$ & $73.7,55-91$ \\
$\quad$ year & M33/F66 & M105/F22 & M73/F14 \\
Gender, M/F & & & \\
Metastatic site & 51 & 32 & 28 \\
$\quad$ Lung & 40 & 47 & 26 \\
$\quad$ Bone & 36 & 43 & 17 \\
$\quad$ Brain & 25 & 44 & 17 \\
$\quad$ Liver & 10 & 36 & 23 \\
$\quad$ Adrenal grand & 13 & 37 & 25 \\
$\quad$ Lymph nodes other & & & \\
$\quad$ than regional nodes & 44 & 31 & 16 \\
$\quad$ Other sites & 41 & 47 & 37 \\
$\quad$ Pleural effusion & & & \\
Non-metastatic & & & \\
Age (median, range), & $73.2,50-88$ & $74.8,56-89$ & $73.8,34-94$ \\
$\quad$ year & & & \\
Gender, Male/Female & M10/F19 & M43/F9 & M130/F17 \\
\hline
\end{tabular}

EGFR: Epidermal growth factor receptor; Adeno: EGFR mutated adenocarcinoma; Squamous: squamous cell cancer; Small: small cell lung cancer.

survival analysis. Clinicopathological characteristics of these patients are shown in Table I.

Metastatic patterns. Patients with EGFR-mutated adenocarcinoma were divided into four clusters: cluster I (26 patients), cluster II (24 patients), cluster III (27 patients), and cluster IV (22 patients; Figure 1A). SCLC patients were divided into four clusters: cluster I (37 patients), cluster II (32 patients), cluster III (20 patients), and cluster IV (38 patients; Figure 1B). Patients with squamous cell carcinoma were divided into four clusters: cluster I (31 patients), cluster II (10 patients), cluster III (17 patients), and cluster IV (29 patients; Figure 1C). There was no difference in patient background among the four clusters of each of the three types of lung cancer, except for gender in patients with EGFR-mutated adenocarcinoma, and age in SCLC patients and those with squamous cell carcinoma. The respective metastatic patterns are shown in Figure 2A-C. The four clusters of metastatic patterns in the three types of lung cancer patients were significantly different $(p<0.001)$.

Survival. The survival curves of the four clusters of metastatic patterns in the three types of lung cancer, and that of patients without metastasis, are shown in Figure 3A-C.

The results of the univariate analysis are shown in Table II. Among patients with cluster I, II, III, and IV, and those without metastasis in each of the three types of lung cancer, there was 
A

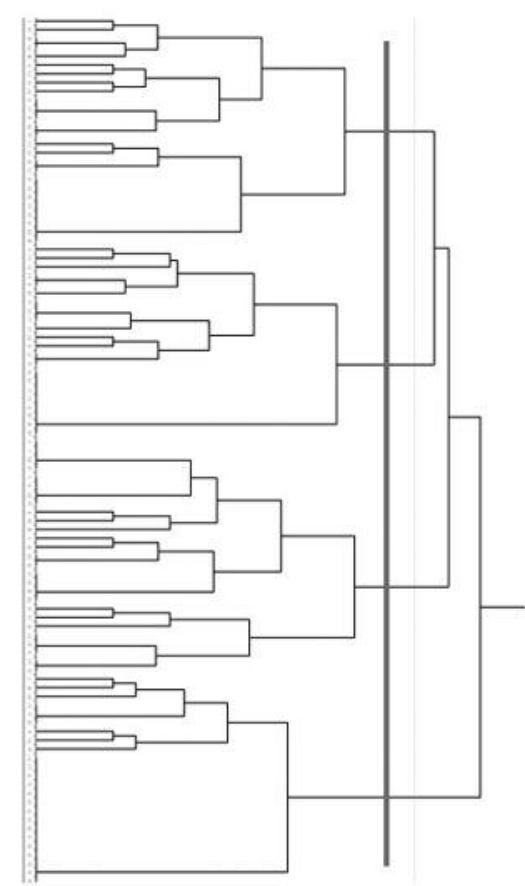

B

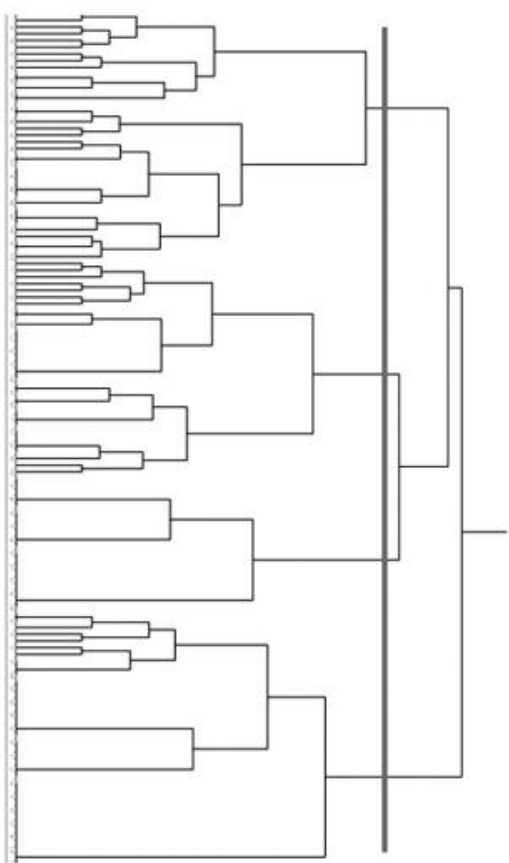

C

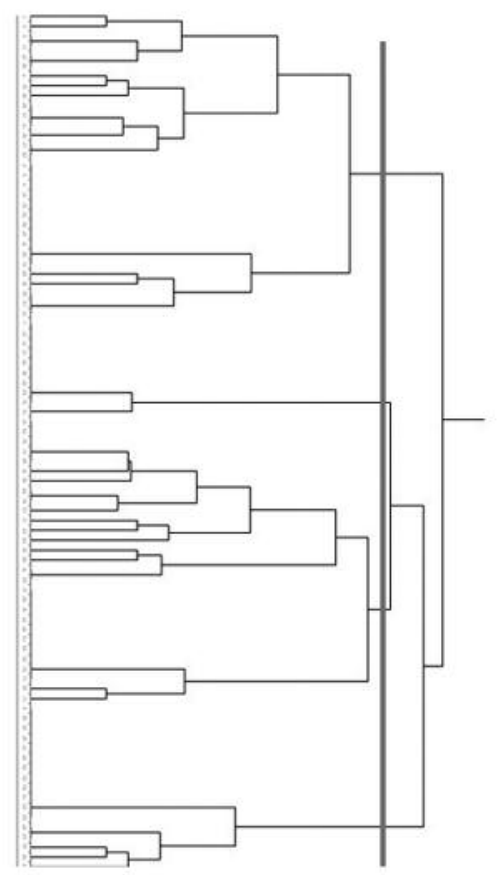

Figure 1. Patients with EGFR mutated adenocarcinoma were divided into 4 clusters: cluster I, cluster II, cluster III, and cluster IV (A). SCLC patients were divided into 4 clusters: cluster I, cluster II, cluster III, and cluster IV (B). Patients with squamous cell carcinoma were divided into 4 clusters: cluster I, cluster II, cluster III, and cluster IV (C). EGFR, Epidermal growth factor receptor (EGFR); SCLC, Small cell lung cancer.

a significant difference in survival $(p<0.001)$. In $E G F R$-mutated adenocarcinoma patients, survival in patients with cluster II was significantly better than those with cluster I, II, and III. In this type of lung cancer, survival in cluster IV (liver-other sites type) was poorer than in clusters I, II, and III. In SCLC patients, survival in cluster IV was poorer than in cluster I, II, and III. However, in patients with squamous cell cancer, no difference in survival was observed between the different clusters.

The results of the multivariate analysis are shown in Table III. In EGFR-mutated adenocarcinoma patients, age, Nfactor, and presence of pleural fluid were unfavorable prognostic factors. On the other hand, being in cluster II was a favorable factor. In SCLC patients, smoking and maximum size of the primary lesion were unfavorable prognostic factors. In addition, being in cluster IV tended to be a poor prognostic factor, although this did not reach statistical significance $(p=0.0694)$. In squamous cell cancer patients, age and $\mathrm{N}$-factor were unfavorable prognostic factors, but being in a particular cluster was not a prognostic factor.

\section{Discussion}

In bone and brain metastases, for example, symptoms such as pain and paralysis reduce the patient's quality of life (13,
14). It is important to control metastasis and not leave sequelae by detecting metastasis early and applying appropriate systemic chemotherapy and local therapy, such as radiation (13). Such multimodal treatment strategies may lead to an improved prognosis. On the other hand, in liver metastasis, even cases with multiple metastases initially proceed asymptomatically (11). Patients with liver metastases have been reported to have a poor prognosis (9, $10,15)$. It is not easy to control liver metastatic lesions, even in the era of immune checkpoint inhibitors (16), and it may be especially difficult to successfully treat recurrent liver metastatic lesions after chemotherapy (15).

Interestingly, a study on the prognosis of patients with lung cancer using cluster analysis has been reported recently (17). This study was carried out to clarify whether there were prognostic factors other than TNM classification. According to the study, among NSCLC patients, "nonsmoking, female, adenocarcinoma" were good prognostic factors other than TNM classification (17). However, these findings were considered indicative of patient groups in which the presence of a driver gene was revealed, such as EGFR-mutated. To the best of our knowledge, no studies have examined the prognosis of lung cancer patients based on metastatic patterns. When examining prognosis using a 

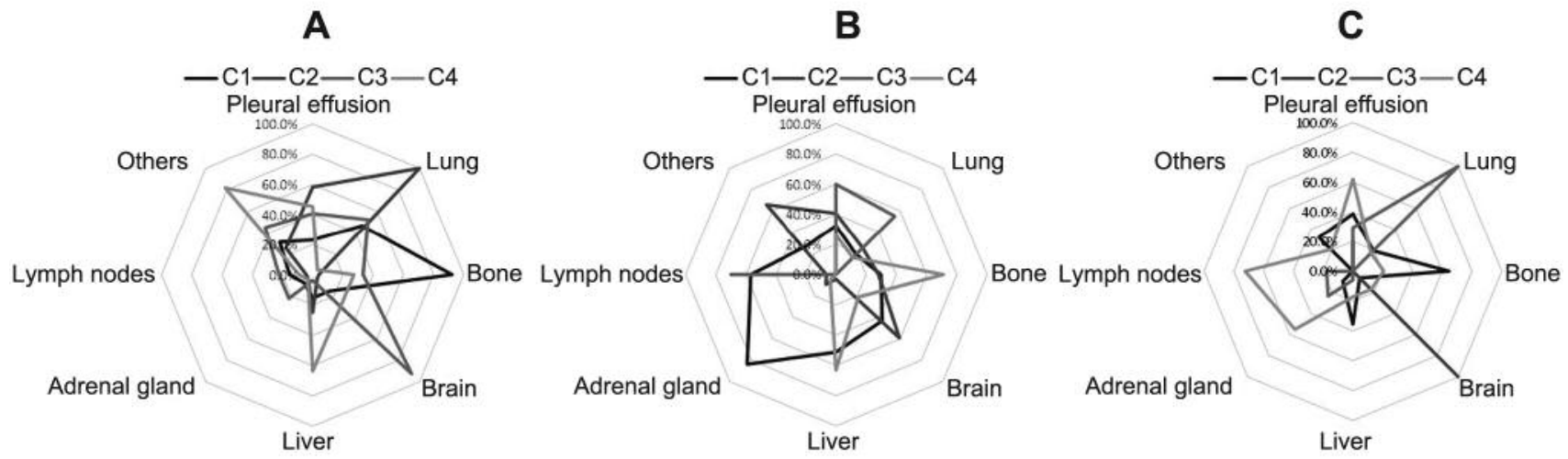

Figure 2. The respective metastatic patterns: Patients with EGFR mutated adenocarcinoma (A), patients with small cell lung cancer (B), and patients with squamous cell carcinoma $(C)$. EGFR, Epidermal growth factor receptor (EGFR).
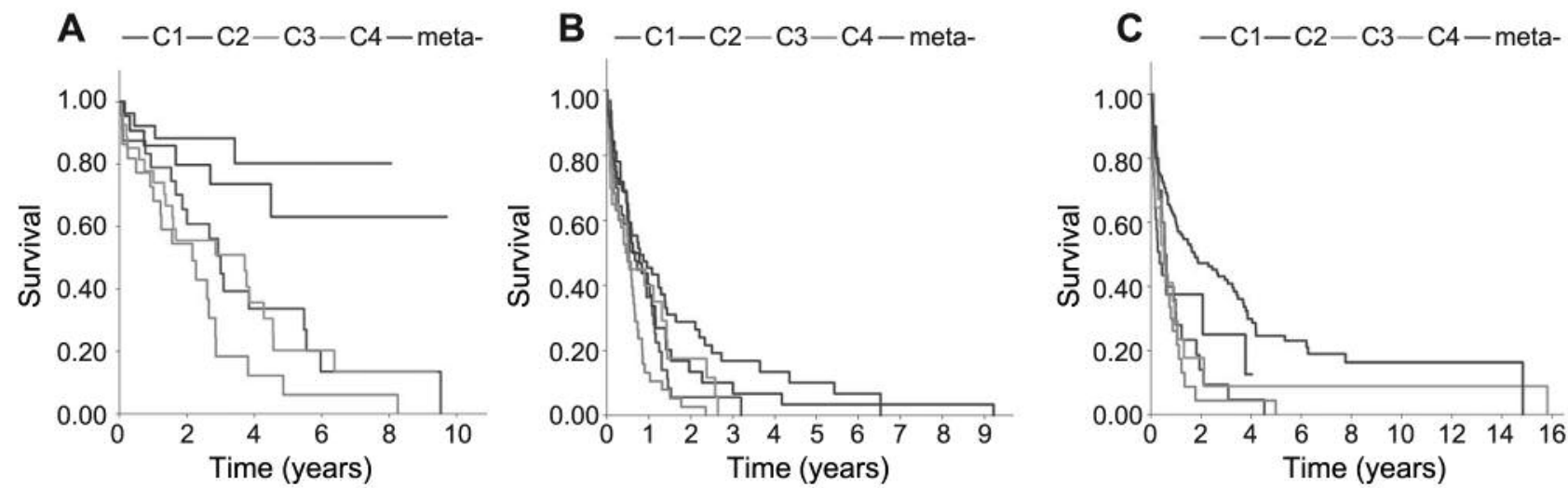

Figure 3. Survival curves of 4 clusters of metastatic patterns in three types of lung cancer patients and that of patients without metastasis: Patients with EGFR mutated adenocarcinoma (A), patients with small cell lung cancer patients $(B)$, and patients with squamous cell carcinoma (C). EGFR, Epidermal growth factor receptor (EGFR).

statistical analysis method, there are two main methods for grouping patients. One is a method of analyzing all patients with a disease together, such as lung cancer as a whole or non-small cell lung cancer as a whole. The other is by dividing patients into groups based on histological type and driver gene. Using the former method, Viadana et al. reported the relationship between metastasis and prognosis in a large number of autopsied adenocarcinoma patients, regardless of the primary site (18). It is important to know which organ metastasis makes the prognosis worse. However, considering that the response to treatment differs depending on the primary site, even for the same adenocarcinoma, it may be easier to interpret the results separately by primary organ, histologic type and driver gene. Therefore, in this study, we examined metastatic patterns and prognosis in each of the three lung cancer subsets divided by tissue type and driver gene.
In this study, the following two points became clear. First, four clusters of metastatic patterns existed in each of the three lung cancer subset groups, and there were significant differences between these clusters of metastatic patterns. Second, in the univariate analysis, a significant difference in survival was identified among the four clusters in metastatic patterns and those without metastasis in each of the three lung cancer types. Additionally, in EGFR-mutated adenocarcinoma patients, 'better survival' was found in cluster II. In SCLC patients, being in cluster IV tended to be a poor prognostic factor $(p=0.0694)$. In squamous cell cancer patients, age and T-factor were unfavorable prognostic factors, but none of the cluster types were significant prognostic factors. Our results suggested that it might be possible to identify groups of patients with good or poor prognosis using metastatic pattern analyses. In squamous cell carcinoma patients, however, the difference was not 
Table II. Comparison results of among each cluster type of lung cancer patients and those without metastasis evaluated with logrank test.

\begin{tabular}{lccc}
\hline Cluster type & \multicolumn{3}{c}{$p$-Value } \\
\cline { 2 - 4 } & Adeno & Small & Squamous \\
\hline Cluster I and cluster II & 0.0132 & 0.3018 & 0.2759 \\
Cluster I and cluster III & 0.7995 & 0.6928 & 0.7397 \\
Cluster I and cluster IV & 0.0688 & 0.13 & 0.9919 \\
Cluster I and patients & 0.0048 & 0.031 & $<0.001$ \\
$\quad$ without metastasis & & & \\
Cluster II and cluster III & 0.0137 & 0.6127 & 0.4775 \\
Cluster II and cluster IV & $<0.001$ & 0.0191 & 0.3587 \\
Cluster II and patients & 0.4162 & 0.3875 & 0.1854 \\
$\quad$ without metastasis & & & \\
Cluster III and cluster IV & 0.1244 & 0.0975 & 0.6239 \\
Cluster III and patients & 0.0023 & 0.1615 & 0.0139 \\
$\quad$ without metastasis & & & \\
Cluster IV and patients & $<0.001$ & $<0.001$ & $<0.001$ \\
$\quad$ without metastasis & & & \\
Cluster I and cluster II+III+IV & 0.8128 & 0.9685 & 0.5564 \\
Cluster II and cluster I+III+IV & 0.0015 & 0.0887 & 0.2922 \\
Cluster III and cluster I+II+IV & 0.5825 & 0.5841 & 0.777 \\
Cluster IV and cluster I+II+III & 0.0045 & 0.0165 & 0.642 \\
\hline
\end{tabular}

Adeno: EGFR mutated adenocarcinoma; Squamous: squamous cell lung cancer; Small: small cell lung cancer.

confirmed in multivariate analysis. It is beyond our knowledge why there were no significant differences among squamous cell carcinoma patients. In squamous cell carcinoma patients, $\mathrm{N}$-factor was a significant prognostic factor. Taking these results into consideration, we supposed that the primary lesion might be more important for prognosis in these patients.

Although this study has provided some novel information, there were certain limitations. Firstly, there was no pathological confirmation of distant metastases due to the diagnostic imaging methods employed. Secondly, although our study included consecutive patients with pathologically proven lung cancer, our small patient base might not reflect the overall patient population in the community. Thirdly, this was a retrospective analysis of only metastatic data from the time of the initial diagnosis of lung cancer, and there was no evaluation of metastases which developed during the clinical course of the disease. The results might be different if we were able to consider data on the metastases that developed during the clinical course. However, the type of diagnostic imaging techniques carried out, and the intervals at which they were performed, may be problematic. Lastly, this was not a study examining the biological mechanism of distant metastasis or evaluating them microscopically. We studied 'clinically' meaningful metastases, and metastases observed in available images, with clinically defined symptoms. Despite these limitations, however, data obtained from
Table III. Multivariate analysis of prognostic factors using Cox's proportional hazard model.

\begin{tabular}{lccc}
\hline Factors & Odds ratio & $95 \%$ confidence interval & $p$-Value \\
\hline Adeno & & & \\
$\quad$ Age & 1.0339 & $1.0025-1.0662$ & 0.0341 \\
Size & 1.0121 & $0.9942-1.0302$ & 0.1859 \\
N-factor & 1.5345 & $1.2067-1.9514$ & $<0.001$ \\
Pleural effusion & 2.4102 & $1.3925-4.1720$ & 0.0017 \\
$\quad$ Cluster II & 0.2513 & $0.1049-0.6017$ & 0.0019 \\
Small & & & \\
$\quad$ Smoking & 0.9995 & $0.9991-0.9998$ & 0.003 \\
Size & 1.0082 & $1.0006-1.0158$ & 0.0334 \\
Cluster IV & 1.4537 & $0.9708-2.1769$ & 0.0694 \\
Squamous & & & \\
Age & 1.0331 & $1.0044-1.0626$ & 0.0236 \\
$\quad$ N-factor & 1.5437 & $1.1365-2.0968$ & 0.0054 \\
Pleural effusion & 1.441 & $0.8761-2.3701$ & 0.1502 \\
\hline
\end{tabular}

Adeno: EGFR mutated adenocarcinoma; Squamous: squamous cell lung cancer; Small: small cell lung cancer.

research approaches such as this may inform molecular biological studies on metastatic patterns.

In this study, we revealed that each of the three lung cancer subset groups had a specific pattern of metastasis. It might become clear that there are patient groups whose metastatic patterns are associated with prognosis. It is worthwhile carrying out diagnostic imaging and treatment in consideration of metastasis patterns.

\section{Conflicts of Interest}

The Authors have no conflicts of interest to declare regarding this study.

\section{Authors' Contributions}

SO, HW, HY, SS, KM and TK had full access to all of the data in this study, and take responsibility for the integrity of the data and the accuracy of the data analysis. SO, HS and NH contributed substantially to the study design, data analysis and interpretation, and writing the manuscript.

\section{References}

1 Ewing $\mathbf{J}$ and Metastasis: Neoplastic Diseases: A Treatise on Tumours. 3rd ed. Philadelphia, PA: W.B. Saunders, pp. 76-88, 1928.

2 Woodhouse EC, Chuaqui RF and Liotta LA: General mechanisms of metastasis. Cancer 80: 1529-1537, 1997. PMID: 9362419. DOI: 10.1002/(sici) 1097

3 Paget S: The distribution of secondary growths in cancer of the breast. Lancet 133: 571-573, 1999. PMID: 2673568.

4 Oikawa A, Takahashi H, Ishikawa H, Kurishima K, Kagohashi $\mathrm{K}$ and Satoh H: Application of conditional probability analysis to distant metastases from lung cancer. Oncol Lett 3: 629-634, 2012. PMID: 22740965. DOI: 10.3892/ol.2011.535 
5 Lanuti M: Surgical management of oligometastatic non-small cell lung cancer. Thorac Surg Clin 26: 287-294, 2016. PMID: 27427523. DOI: $10.1016 /$ j.thorsurg.2016.04.002

6 Eberhardt WE, Mitchell A, Crowley J, Kondo H, Kim YT, Turrisi A 3rd, Goldstraw P and Rami-Porta R: The IASLC Lung Cancer Staging Project: Proposals for the revision of the M descriptors in the forthcoming eighth edition of the TNM Classification of Lung Cancer. J Thorac Oncol 10: 1515-1522, 2015. PMID: 26536193. DOI: 10.1097/JTO.0000000000000673

7 Shahidi $\mathrm{H}$ and Kvale PA: Long-term survival following surgical treatment of solitary brain metastasis in non-small cell lung cancer. Chest 109: 271-276, 1996. PMID: 8549197. DOI: 10.1378/chest.109.1.271

8 Kodama T, Kikuchi N, Satoh H and Ohtsuka M: Metacarpal bone metastasis from lung cancer. Onkologie 32: 216-217, 2009. PMID: 19372720. DOI: 10.1159/000203347

9 Nakazawa K, Kurishima K, Tamura T, Kagohashi K, Ishikawa $\mathrm{H}$, Satoh $\mathrm{H}$ and Hizawa N: Specific organ metastases and survival in small cell lung cancer. Oncol Lett 4: 617-620, 2012. PMID: 23205072. DOI: 10.3892/ol.2012.792

10 Tamura T, Kurishima K, Nakazawa K, Kagohashi K, Ishikawa $\mathrm{H}$, Satoh $\mathrm{H}$ and Hizawa N: Specific organ metastases and survival in metastatic non-small-cell lung cancer. Mol Clin Oncol 3: 217-221, 2015. PMID: 25469298. DOI: 10.3892/mco. 2014.410

11 Kagohashi K, Satoh H, Ishikawa H, Ohtsuka M and Sekizawa $\mathrm{K}$ : Liver metastasis at the time of initial diagnosis of lung cancer. Med Oncol 20: 25-28, 2003. PMID: 12665681. DOI: 10.1385/MO:20:1:25

12 Ball GH and Hall DJ: A clustering technique for summarizing multivariate data. Behav Sci 12: 153-155, 1967. PMID: 6030099. DOI: $10.1002 /$ bs. 3830120210

13 Ampil FL and Baluna R: Is "routine" cranial irradiation in hemiplegic lung cancer patients with brain metastases justified? J Palliat Med 13: 794-795, 2010. PMID: 20636143. DOI: $10.1089 /$ jpm.2010.0028
14 Nakagawa K, Ohkuma K, Yamashita H, Masuda M, Matsumoto $\mathrm{Y}$ and Gotoh T: Radiation therapy did not alleviate complete paralysis due to metastasis of lung adenocarcinoma to thoracic vertebrae until four months later. Acta Oncol 50: 606-608, 2011. PMID: 21250927. DOI: 10.3109/0284186X.2010.550314

15 Ardizzoni A, Tiseo M and Boni L: Validation of standard definition of sensitive versus refractory relapsed small cell lung cancer: a pooled analysis of topotecan second-line trials. Eur J Cancer 50: 2211-2218, 2014. PMID: 24981975. DOI: 10.1016/ j.ejca.2014.06.002

16 Shiroyama T, Suzuki H, Tamiya M, Tamiya A, Tanaka A, Okamoto N, Nakahama K, Taniguchi Y, Isa SI, Inoue $\mathrm{T}$, Imamura F, Atagi $\mathrm{S}$ and Hirashima T: Clinical characteristics of liver metastasis in nivolumab-treated patients with non-small cell lung cancer. Anticancer Res 38: 4723-4729, 2018. PMID: 30061241. DOI: 10.21873 /anticanres.12779

17 Sakr L, Small D, Kasymjanova G, Suissa S and Ernst P: Phenotypic heterogeneity of potentially curable non-small-cell lung cancer: cohort study with cluster analysis. J Thorac Oncol 10: 754-761, 2015. PMID: 25898956. DOI: 10.1097/JTO. 0000000000000505

18 Viadana E and Au KL: Patterns of metastases in adenocarcinoma of man. J Med 6: 1-14, 1997. PMID: 1056412.
Received December 2, 2019

Revised December 12, 2019 Accepted December 13, 2019 